\title{
Assessing the Hair Colourings' Impact on Quality of Life
}

Beresniak $A^{1,2^{*}}$, Jean-Paul Auray ${ }^{3}$, Duru $\mathbf{G}^{3}$, Krueger GG ${ }^{4}$, Talarico $\mathbf{S}^{5}$, Aractingi $\mathbf{S}^{2}$, Tsutani $K^{6}$, Dupont $\mathbf{D}^{7}$ and de Linares $\mathbf{Y}^{8}$

${ }^{1}$ Data Mining International, Geneva, Switzerland

${ }^{2}$ Paris-Descartes University, Paris, France

${ }^{3}$ Cyklad Group, Lyon, France

${ }^{4}$ University of Utah, Salt Lake City, USA

${ }^{5}$ Federal University of Sao Paulo, Sao Paulo, Brazil

${ }^{6}$ University of Tokyo, Tokyo, Japan

${ }^{7}$ Data Mining America, Montreal, Canada

${ }^{8}$ L'Oréal Research \& Innovation, Clichy, France

"Corresponding author: Beresniak A, Route de l'Aéroport, 29-31, CP 221, CH-1215, Geneva 15, Switzerland, Tel: +41227993400; Fax: +41227883850; E-mail: aberesniak@datamining-international.com

Received date: October 07, 2015; Accepted date: December 12, 2015; Published date: December 17, 2015

Copyright: $\odot 2015$ Beresniak A, et al. This is an open-access article distributed under the terms of the Creative Commons Attribution License, which permits unrestricted use, distribution, and reproduction in any medium, provided the original author and source are credited.

\begin{abstract}
Objective: Research shows that physical appearance is one of the key drivers of personal well-being. An improvement in facial attractiveness is associated with positive changes in emotional and social dimensions of one's life. Hair colouring is an important means of cosmetic improvement in women and men. However, no research has been carried out so far to assess its impact on quality of life (QoL) using an instrument validated for cosmetic products and physical appearance. The main objective of this study was to assess the impact on QoL of two hair colouring products (Excellence ${ }^{\circledR}$ by L'Oréal Paris and Olia ${ }^{\circledR}$ by Garnier) using the BeautyQoL ${ }^{\circledR}$ questionnaire, specially designed and internationally validated for use in cosmetology.
\end{abstract}

Methods: A randomized control study has been carried out involving a total of 240 adult women (45-70 years old) assigned in two groups "Excellence" and "Olia". The BeautyQoL ${ }^{\circledR}$ questionnaire was administered in order to assess the impact of the two hair colouring treatments on QoL across five well-being dimensions: social life, self-confidence, mood, energy, and attractiveness. At the end of the study, subjective satisfaction questionnaires were used to assess the perceived performance and tolerability for each product, as well as interest in future use.

Results: The results of this study suggest that the QoL was improved in subjects using hair colouring products based on the overall and dimension-specific BeautyQoL ${ }^{\circledR}$ scores at the end of the experiment. The results of the satisfaction questionnaire were also conclusive, confirming the subjects' good tolerability and overall satisfaction of the tested hair colouring products.

Conclusion: Hair colouring is used by millions of women and men who wish to look good, enhance their appearance, self-confidence and their overall attractiveness. For the first time, using the internationally validated BeautyQoL ${ }^{\circledR}$ questionnaire developed to specifically assess cosmetic products and physical appearance, the results of this study firmly establish that hair colouring contributes to improving the overall and dimension-specific QoL scores, and the well-being of users worldwide.

Keywords: BeautyQoL; Hair colouring

\section{Introduction}

In today's society, preserving a young and good healthy appearance is very much valued. Having a nice overall appearance influences the self-confidence, mood, overall appeal and social acceptance. Attractiveness involves both how people think about themselves as well as their behaviour toward others [1]. Given that a person's physical attractiveness is a major factor in his or her life experiences, it has an impact on one's self-esteem. The research shows that improving a physical trait improves attitude, personality, and self-perception. Furthermore, improving physical attractiveness is associated with positive changes in the emotional and social dimensions of one's life, thus improving interpersonal interactions and relationships [2,3,4].

Conversely, idiosyncratic physical or facial traits, such as noticeable skin conditions or discolorations (such as vitiligo), or presenting a neglected physical appearance can lead to social stigma and detrimental psychosocial effects, and there with the risk of negatively impacting one's self-image, relationships, social and professional status, and quality of life (QoL) $[5,6]$. Because cosmetic products are designed to enhance appearance, they are expected to improve people's selfperception, their relationships and their QoL.

The hair coloration means changing the hair colour, the main reason for this practice is cosmetic (e.g. to cover grey hair, to change to a colour regarded as more fashionable or desirable, or to restore the 
Page 2 of 6

original hair colour after it had been dyed or discoloured from bleaching processes). With advances made in the composition of hair coloration products and in the techniques used, hair colouring is immensely popular and enables people to enhance their appearance. Today, one woman out of two and one man out of ten, use hair coloration. In the USA, over 75 percent of women alter their hair colour [7]. This demonstrates a need to hide white hair, change the natural shade (fully or partially) in a sustainable or ephemeral way, revive natural colour, or accentuate reflections. By improving their appearance with hair coloration, people are more confident and attractive and feel good about themselves, the consequence of which is the improvement in their self-image and their overall well-being.

Measuring QoL entails a multidimensional assessment of physical, social, psychological, and emotional realms. By assessing and quantifying the effects of cosmetic products on these dimensions, the improvement in QoL can be quantified and better understood [1].

Many QoL instruments have been developed over the years. Some instruments are generic, such as the Short Form 36 (SF-36), a multipurpose health survey comprising 36 questions [8]. The SF-36 has proven useful in surveys of general and specific populations for assessing and comparing the relative burden of some conditions, and for differentiating the health benefits produced by a wide range of treatments and interventions. Other instruments have also been developed and used to specifically assess the QoL with regards to some conditions or diseases $[9,10,11]$.

Considering constant research efforts for the development of safe and effective high quality cosmetic products, and their huge utilisation worldwide, the development of robust QoL assessment methods and validated instruments will contribute to establishing strong evidence for the benefits and value of cosmetic products [4].

The BeautyQoL ${ }^{\circ}$ questionnaire is a new instrument which has been developed to address this unmet need; specifically assessing cosmetic products on physical appearance. The BeautyQoL ${ }^{\circ}$ scientific instrument assesses five QoL dimensions: social life, self-confidence, mood, energy, and attractiveness. Importantly, the reliability, reproducibility, and robustness of the BeautyQoL instrument have been validated internationally in 13 countries and in 16 languages [3].

As a result, and for the first time, this study proposes to use the BeautyQoL instrument to assess the QoL in users of hair colour treatments. The main objective of this study was to assess and compare the impact on QoL of two hair colouring products using the BeautyQoL questionnaire, developed and validated for use in cosmetology.

\section{Methods}

This open randomized control trial was carried out in France and was designed recruit a total of 240 female subjects to assess and compare the QoL impact of two hair colouring products: Excellence by L'Oréal (EX) and Olia by Garnier (OL). The specific colouring products are described (Table 1).

\begin{tabular}{|l|l|}
\hline Excellence & OLIA \\
\hline Very light golden & Very light golden blond \\
\hline blond & Blond \\
\hline Auburn & Intense dark red blond \\
\hline
\end{tabular}

\begin{tabular}{|l|l|}
\hline Light brown golden & Light brown golden \\
\hline Light brown & Light brown \\
\hline
\end{tabular}

Table 1: Hair Colouring Products Tested.

A minimum of 120 subjects were assigned to Group 1 (EX) and to Group $2(\mathrm{OL})$. The inclusion and exclusion criteria are described in Table 2 and Table 3, respectively. The data collection was conducted during the last quarter 2012. Only the use of regular shampoo was allowed on the hair and scalp, and no excessive exposure to sunlight or UV was permitted during the study timeframe. Considering that both tested products are marketed, this study did not raise any ethical issue. According to the French Law of August 9th 2004, no submission to ethic committee was mandatory (Table 2 and 3 ).

\section{General Criteria}

Good general-health

Were informed of the objectives of the study and provided express written consent

Participating subjects were warned of the need for and of the duration of controls in order to expect a perfect adherence to the protocol established by the clinical trial centres.

\section{Specific Criteria}

Caucasian subjects

Gender: female

Between 45 and 70 years

Phototype: I to IV

Subjects making permanent colorations at home only, or sometimes at home and sometimes at a hair salon

Subjects making their own hair colouring

Subjects who used Excellence Pro-Keratin ${ }^{\circledR}$ L'Oréal Paris at least 3 times in the last 12 months

Subjects performing their hair colouring less than 4 months apart

Shade of colour used or considered:

Very light golden blond

Blond

Dark Blonde Intense Red / Auburn

Light Golden Brown

Light Brown

Subjects had their last hair colouring at least 4 weeks prior to inclusion

Subjects needed to have white or gray hair, that was quite visible or very visible prior to performing hair colouring

Subjects could buy their own hair colouring or could be purchased by a third party with instructions

\section{Table 2: Inclusion Criteria.}

Compliance to the study protocol was monitored and in the case of non-compliance, subjects were excluded from data-analysis. Each subject completed a daily diary to record on which days the hair colouring products were being used, and to record the use of any medical treatment or medicines.

At day $1\left(\mathrm{~T}_{0}\right)$, the subjects coming for evaluation did so without having applied any colouring product on their hair and scalp from the night before for at least 12 hours. They had read, signed and dated the information sheet (instructions on the use of hair colouring products 
and restrictions related to the study), and the consent form. These documents were also signed and dated by the person in charge of verification process. Thereafter a study technician collected the data using the BeautyQoL' questionnaire.

Pregnant or breast feeding women or planning to become pregnant during the
study
Cutaneous pathology on the measurement zone (eczema, etc. )
Intense itching or significant flaking on the scalp
Very sensitive scalp
History of allergy to a dye product
Participation in another clinical trial during the period of the study
Subjects having performed hair colouring with henna or with progressive hair
colouring
Subjects having done bleaching, lightening, straightening or a permanent within
15 days prior to inclusion
Subjects working in a field related to beauty care, cosmetology, aesthetics or
hairdressing, journalism or advertising, marketing or market research
Immunosuppressed subjects
History of skin cancer
Subjects who underwent surgery under general anaesthesia in the month
preceding the start of the study
Subjects who started, modified or ceased hormone therapy in less than 6 weeks
prior to the start of the study
Employees of the clinical research organization

Table 3: Exclusion Criteria.

The BeautyQoL ${ }^{\circ}$ questionnaire (French version 3.0), "Quality of Life at $\mathrm{T}_{0}$ ", was self-administered. The purpose of collecting the BeautyQoL results at $\mathrm{T}_{0}$ was only to assess the comparability and homogeneity between the two subject groups.

The hair colouring products were distributed to the subjects using a randomization protocol. The subjects received a daily diary for recording any side effects and intolerances during the study.

The same day, the subjects performed a skin allergy test at home by applying (with a cotton swab) a small quantity of the hair colouring product behind the right ear. The subjects were notified by the study technician of the instructions to be used during the 48 hours after first applying the test product: namely not to wash their hair, and not to remove the product from the skin allergy test area in any way until the second visit. In case of low intensity itching, the subjects were instructed not to touch or scratch the area where the product had been applied. In case of a severe reaction, subjects were instructed to rinse the skin test area thoroughly, to shampoo their hair and by inspecting the skin test area behind the ear, and to notify the study centre as soon as possible.

At day 2, the subjects checked at home for the presence or absence of skin test reaction and/or signs of irritation and/or discomfort on the area where the product had been applied. In the absence of a reaction, the subjects were instructed to do their hair colouring using the product distributed at day 1 , according to the instructions provided in the product packaging.

At day $3\left(\mathrm{~T}_{3}\right)$, the subjects were seen at the study centre and returned their daily diary and the remaining product. The technician collected the data from the BeautyQoL ${ }^{\circ}$ questionnaire "Quality of life $\mathrm{T}_{3}$ ". The subjects also completed the satisfaction questionnaire relevant to the attributes of the study product used.
The BeautyQoL' questionnaire includes a total of 42 questions that address five QoL dimensions, categorized under: social life, selfconfidence, mood, energy and attractiveness. The questionnaire was administered to the subjects of both groups G1 (EX) and G2 (OL) at the end of the experiment (day 3) to assess if the hair colouring products tested and the resulting physical appearance had had an impact on their QoL.

The study was conducted in accordance with the Declaration of Helsinki (1964) and amended in accordance with the principles of Good Clinical Practice CPMP / ICH / 135/95.

Each subject was verbally informed of the study protocol and signed an informed consent form.

\section{Results}

\section{Demographics and subject's description}

A total of 119 subjects were recruited and randomized to Excellence (Ex), and 121 subjects were randomized to Olia $(\mathrm{OL})$ hair colouring products, respectively. After excluding the 2 and 6 subjects who did not show up at evaluation in each group (plus 2 subjects who were counted twice in the first group), the analysis included 115 subjects in each group.

The overall duration of this study was between 4 and 11 days. Relevant to the test of equality of means, there was no statistical difference between the mean duration of the experiment between the two groups, namely: 5.27 days $[\mathrm{SD}=1.416](\mathrm{EX})$, and 5.17 days [SD=1.311] (OL), $\mathrm{p}=0.563$.

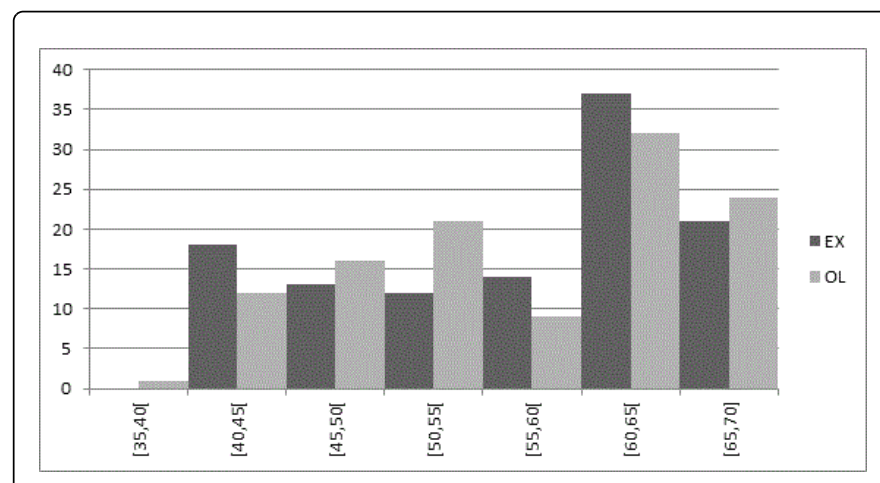

Figure 1: Age Distribution (from 35 to 70) by 5 Year Intervals of the Subjects Randomized to Each Product. EX: Excellence by L'Oréal Paris, OL: Olia by Garnier.

The age of the subjects in each group is represented in Figure 1, with a mean age of 56.5 years for both groups (Table 4 ). The work place and type of work was similar for the two groups.

\begin{tabular}{|l|l|l|l|l|}
\hline & N & Mean & Std. Deviation & Median \\
\hline EX & 115 & 56.41 & 9.095 & 60.00 \\
\hline OL & 115 & 56.54 & 8.685 & 59.00 \\
\hline Total & 230 & 56.47 & 8.873 & 59.00 \\
\hline
\end{tabular}

Table 4: Mean age of participating subjects.

The subjects from both groups reported having hair that was: 
Page 4 of 6

Very oily (1\%), oily (11\%), normal (42\%), dry (35\%), very dry (4\%), and mixed (7\%).

Very short $(8 \%)$, short $(41 \%)$, shoulder length $(42 \%)$, long $(8 \%)$, or very long (1\%).

Very fine $(12 \%)$; fine $(37 \%)$; average $(27 \%)$; thick $(21 \%)$ or very thick (3\%).

Straight hair (34.3\%), soft slightly waved hair (40\%), wavy (14\%), curly $(5.2 \%)$, very curly $(6 \%)$, or frizzy $(<1 \%)$

For both groups, $63 \%$ of respondents reported washing their hair 2-3 times a week, $30 \%$ once a week, $6 \%$ every day and $<1 \%$ less frequently than once weekly. Both groups were alike relative to the hair diameter; subjects reported thick (33.4\%), normal (46.1\%), or thin and sparse hair (20.4\%). Relevant to the condition of their scalp, subjects from both groups reported having normal $(70 \%)$, oily (14.3\%) or a dry scalp (15.6\%). Lastly, $1 \%$ reported having lots of dandruff, $15.2 \%$ reported some and $84 \%$ none.

A similar proportion of subjects, in both groups, reported being married or living as a couple (48\%), the remaining being either single, widows, or divorced (52\%).

The use and type of hair colouring techniques in the last 12 months (at home or at a hair salon) were also similar between the EX and OL groups, with $100 \%$ of the subjects in both groups having used permanent colouring products with visible re-growth, $14.8 \%$ (EX) and $12.2 \%(\mathrm{OL})$ used tone on tone colour that lasts about 6 weeks, $1.7 \%$ in both groups used hair bleaching or lightening, and 14.8\% (EX) and $13.9 \%$ (OL) used "light locks" or hair highlights.

In the previous 12 months, the majority of subjects had performed their hair coloration at home only, either by themselves or assisted by another person, in the following proportions: 67\% (EX) and 64.3\% $(\mathrm{OL})$, versus $33 \%(\mathrm{EX})$ and $35.7 \%(\mathrm{OL})$ who had their coloration performed sometimes at home and sometimes at a hair salon. Concerning their previous use in the past 12 months, the proportion was also similar between the two groups, with $23.5 \%$ (EX) vs. $24.3 \%$ $(\mathrm{OL})$, and $76.5 \%(\mathrm{EX})$ vs. $75.7 \%(\mathrm{OL})$, who had dyed their hair three times, and more than three times, respectively.

Both groups also had similar hair colouring use and frequency with $100 \%$ of subjects in both groups having used the product alone. $100 \%$ of subjects had their last hair colouring done (at home or at the hair salon) $\geq 4$ weeks prior to completing the screening interview. In the previous 12 months, 28 subjects (EX) and 35 subjects (OL) reported having their hair dyed at a hair salon every 3-4 weeks, 61 (EX) and 58 (OL) at every 5 weeks to 8 weeks, and 26 (EX) and $22(\mathrm{OL})$ at every 10 weeks or more, respectively.

All subjects in both groups reported i) having no history of allergic reactions to hair colouring, and ii) not being pregnant at the time of the screening interview. Similar proportions of subjects reported having a slightly sensitive scalp, namely $16.5 \%$ (EX) and $14.8 \%(\mathrm{OL})$, while $11.3 \%(\mathrm{EX})$ and $7 \%(\mathrm{OL})$ reported having mild itching or flaking of the scalp.

There was a similar distribution of subjects who reported having totally white or grey hair in both groups, namely $10.4 \%$ (EX) and $11.3 \%(\mathrm{OL})$, while $35.7 \%(\mathrm{EX})$ and $36.5 \%(\mathrm{OL})$ reported having a lot of, $33 \%$ (EX) and $25.2 \%$ (OL) some, and 20.9\% (EX) and 27\% (OL) a little. A majority of subjects in both groups responded that usually, their white hair was quite visible at the time of doing their hair colouring.
Regarding hair colouring products used at home, $99.1 \%$ of subjects in both groups indicated that they would typically purchase their products themselves, leaving only $0.9 \%$ who would instruct another person to purchase their products for them.

Regarding the qualitative attributes of the reference product (Excellence by L'Oréal Paris), there was no appreciable difference in the responses provided by the two groups (EX) and (OL). The majority of respondents in both groups respectively agreed to the following statements for the reference product, namely: that it leaves the hair shiny, it perfectly covers all the white hair, it gives a beautiful colour, it gives nice highlights to the hair, it does not irritate the scalp, it leaves the hair soft to the touch, it has a pleasant odour during pause time, it gives a uniform colour from root to tip, it is fast to use, it gives a natural colour (not artificial), it gives a beautiful long lasting colour, it does not damage the hair, it is easy to use and rinse, it is fast to prepare, it does not drip during the pause time, it is easy to spread on the hair, it has a pleasant odour during application, it takes care of the hair, it makes the hair stronger and more resistant, it is gentle on the scalp. In general, most subjects were either very satisfied (52\%) or satisfied (46\%) with either product, the remaining $2 \%$ being neutral.

\section{Quality of Life}

Under the BeautyQoL' scientific protocol, the 42 questions are used to derive i) an overall QoL score, ii) five sub scores for each specific dimension (D1, D2, D3, D4, D5). All algorithmic scores are comprised between 0 (worst) and 100 (best). The corresponding results from the BeautyQoL questionnaire are presented below for Group 1 (EX) and Group 2 (OL), using: i) the overall score, and ii) the composite scores, before and after the experiment, at $\left(\mathrm{T}_{0}\right)$ and $\left(\mathrm{T}_{3}\right)$ respectively.

The scores of the tests performed to compare the equality of means are provided in Table 5. The results do not reject the hypothesis of equality of mean scores between the two groups, and this, no matter what the scores are. This confirms that the two groups are homogeneous concerning their QoL before starting the study (Table $5)$.

\begin{tabular}{|l|l|l|l|l|}
\hline & & N & Mean & Std Deviation \\
\hline GLOBAL Score & $1 \mathrm{EX}$ & 115 & 63,5 & 19,8 \\
\cline { 2 - 5 } & $2 \mathrm{OL}$ & 115 & 62,6 & 20,3 \\
\hline Dimension 1: Social Life & $1 \mathrm{EX}$ & 115 & 57,0 & 22,5 \\
\cline { 2 - 5 } & $2 \mathrm{OL}$ & 115 & 56,0 & 22,7 \\
\hline Dimension 2: Self-Confidence & $1 \mathrm{EX}$ & 115 & 74,3 & 16,2 \\
\cline { 2 - 5 } & $2 \mathrm{OL}$ & 115 & 73,7 & 17,8 \\
\hline Dimension 3: Mood & $1 \mathrm{EX}$ & 115 & 63,3 & 23,5 \\
\hline \multirow{2}{*}{ Dimension 4: Energy } & $2 \mathrm{OL}$ & 115 & 62,5 & 23,5 \\
\hline \multirow{2}{*}{ Dimension 5 : Attractiveness } & $1 \mathrm{EX}$ & 115 & 57,4 & 26,0 \\
\hline & $2 \mathrm{EL}$ & 115 & 57,5 & 25,3 \\
\hline & $2 \mathrm{OL}$ & 115 & 68,1 & 21,0 \\
\hline & & & 18,8 \\
\hline
\end{tabular}

Table 5: Algorithmic BeautyQol Scores (CS) at $\mathrm{T}_{0}$. 
Page 5 of 6

The mean value obtained for each of the six algorithmic composite scores (global and per QoL dimension) for the two groups, Group 1 (EX) and Group $2(\mathrm{OL})$ at $\mathrm{T}_{3}$, is provided, confirming an improvement of QoL in both groups (Table 6).

\begin{tabular}{|l|l|l|l|l|}
\hline & & N & Mean & Std. Deviation \\
\hline \multirow{2}{*}{ GLOBAL score } & $1 \mathrm{EX}$ & 115 & 57,5 & 23,0 \\
\cline { 2 - 5 } & $2 \mathrm{OL}$ & 115 & 56,0 & 22,6 \\
\hline Dimension 1: Social Life & $1 \mathrm{EX}$ & 115 & 52,0 & 24,8 \\
\cline { 2 - 5 } & $2 \mathrm{OL}$ & 115 & 49,6 & 23,5 \\
\hline Dimension 2: Self - Confidence & $1 \mathrm{EX}$ & 115 & 66,8 & 20,8 \\
\cline { 2 - 5 } & $2 \mathrm{OL}$ & 115 & 66,4 & 21,5 \\
\hline Dimension 3: Mood & $1 \mathrm{EX}$ & 115 & 58,0 & 26,2 \\
\cline { 2 - 5 } & $2 \mathrm{OL}$ & 115 & 56,0 & 26,0 \\
\hline \multirow{2}{*}{ Dimension 4: Energy } & $1 \mathrm{EX}$ & 115 & 51,6 & 26,4 \\
\cline { 2 - 5 } & $2 \mathrm{OL}$ & 115 & 51,6 & 25,9 \\
\hline \multirow{2}{*}{ Dimension 5: Attractiveness } & $1 \mathrm{EX}$ & 115 & 62,7 & 22,6 \\
\cline { 2 - 5 } & $2 \mathrm{OL}$ & 115 & 61,3 & 24,1 \\
\hline
\end{tabular}

Table 6: Algorithmic QoL scores at $\mathrm{T}_{3}$.

In order to assess the potential significance of the improvement of the QoL at $\mathrm{T}_{3}$, the scores of the Group1 (EX) and Group 2 (OL) were compared at $\mathrm{T} 3$ with the scores of a theoretical reference population in which each individual would have provided the answer that there were no improvement in any of the 42 questions of the BeautyQoL questionnaire.

The score "Not at all" being equal to 20, considering an average score Savg for the study population, it would then translate to: H0: "Savg $\geq 20$ " versus H1: " Savg $<20$ ".

After testing this assumption for all 42 questions of the BeautyQoL ${ }^{\circ}$ questionnaire and comparing with the composite scores of Group 1 (EX) and Group 2 (OL), H0 cannot be rejected, confirming the statistical significance $(\mathrm{p} \leq 0.05)$ of the improvement of the Qol observed in the two groups.

\section{Satisfaction Questionnaire Results}

The majority of subjects in both groups responded similarly to the effect that they were very satisfied $(57.4 \%)$ or fairly satisfied $(38.3 \%)$ with the product used, only few being not so satisfied (4.3\%). Women in Group 1 (EX) were significantly more likely to be "very satisfied" and less likely to be "somewhat satisfied" or "not very satisfied" compared to the subjects Group $2(\mathrm{OL})$. With reference to the Excellence product (refer to the inclusion criteria), the responses were similar in both groups where subjects responded that the product used was very different $(9.1 \%)$, quite different $(20.9 \%)$, not so different $(57.0 \%)$, and not at all different (13\%). However, the subjects in Group 1 (EX) were significantly more likely to respond that the hair colouring product tested was "not much" or "not at all" different compared to the subjects in Group $2(\mathrm{OL})$ who were more likely to think that the product tested was "very" and "somewhat" different.
In both groups, the vast majority $(>60 \%)$ fully agreed that the product used had left the hair shiny and soft to the touch, perfectly covered all white hair, gave a beautiful colour and nice highlights on the hair, was easy and fast to use, easy to rinse, did not drip during pause time, and was easy to spread on the hair.

Relevant to hair brightness, the answers differed significantly for the two groups: the subjects in Group 1 (EX) were significantly more likely to consider that the product tested was better, and less likely to consider that their usual reference product was better than those in group 2 (OL). Responses also differed significantly for the two groups in terms of coverage of white hair: the subjects in Group 1 (EX) were significantly less likely to believe that the product tested was better than their usual reference product, while it was the opposite for the subjects in Group 2 (OL). Also, more subjects in Group 1 (EX) fully agreed that the product was fast to prepare (82\%) compared to $(70 \%)$ in Group 2 (OL).

Relevant to the odour, the subjects in Group 2 (OL) were more likely to think that the product they had just used had performed better. In both groups, the majority of subjects very much liked their obtained hair colour and reported that it was just right. The vast majority (88\%) answered that the product used had given just the right amount of highlights on their hair. A majority of subjects in both groups indicated that the product tested had covered all their white hair similar to their usual hair colouring products.

\section{Discussion}

In a society where beauty standards have dramatically evolved over the years and now are much valued and promoted in all aspects of our lives, there is social pressure to preserve a young-looking, healthy and beautiful appearance. In parallel, research has shown that physical appearance is one of the key drivers of personal well-being because it influences self-perception and social relations.

Among the many procedures and cosmetic interventions available today to reduce the visible effects of ageing, hair colouring is one of the most popular means of cosmetic improvement for both women and men. The advancements achieved in hair colouring technologies have led to the development of hair colouring treatments that are highly effective, safe, and easy-to-use. Nowadays, there are multiple options and combinations that consumers can consider, as well as a variety of colour nuances that provide very natural looking results. Until now however, no study had been carried out to specifically assess the impact of hair colouring on the physical appearance and QoL.

Accordingly, the main interest and novelty of this randomized open design study is that it uses a robust scientific questionnaire which has been specially designed, tested and validated internationally to assess cosmetic products and physical appearance [3]. Compared to a generic QoL questionnaire (such as the SF-36) that is a multi-purpose health survey for assessing QoL regardless of the populations and conditions, when available, it is preferable and more advisable to use conditionspecific QoL instruments that can achieve more precise results. Consequently, and for the first time, this study assesses the impact of two hair colouring products on the overall well-being with the use of the BeautyQoL ${ }^{\circ}$ specific instrument to derive an overall QoL score, as well as five QoL dimensions: social life, self-confidence, mood, energy, and attractiveness, at the end of the experiment.

Moreover, using a distinct satisfaction subjective questionnaire, this study also assesses the overall satisfaction and personal preferences of 
hair colouring users with regards to the characteristics of the products tested, as well as their respective performance and their tolerability. The results confirm that hair colouring had positively, favourably and similarly influenced the overall QoL scores as well as dimensionspecific composite scores at day 3 in both groups (EX) and (OL).

A limitation of the BeautyQoL questionnaire is the difficulty in comparing QoL scores over time with T0. This is because the structure of the BeautyQoL ${ }^{\oplus}$ instrument allows to measure a difference in QoL (each question asks for a potential improvement), but not to measure a QoL status. In subjective assessments such as are required for personal opinion we and others generally assume that the subject has relatively robust recall, i.e., what it was at T0; BeautyQoL ${ }^{\circ}$ does not and was not designed to provide an objective measure. Nonetheless, an assessment at $\mathrm{T}_{0}$ is very important to test the homogeneity of the groups, thus reducing any potential interpretation bias.

In addition, BeautyQoL ${ }^{\circ}$ scores were compared to those which could have been obtained from virtual subjects who would have provided the worst possible score ("not at all") to each of the 42 questions of the BeautyQoL ${ }^{\circ}$ questionnaire. This additional analysis further validates that all the BeautyQoL ${ }^{\circ}$ scores generated in the study are equal or greater than those derived from considering an hypothetical reference population which would have provided the worst possible answer to each of the 42 questions of the BeautyQoL questionnaire.

Another limitation of this study include the relatively short duration of the experiment considering that users of hair colouring treatments sometimes need time, (days) after their hair coloration to more suitably assess its impact and long-lasting effect, and/or the time to adjust to their new hair colour. Also considering that the reproducibility of the BeautyQoL questionnaire had been validated at 8 days, a longer duration study could have potentially influenced the results in both groups. As the study subjects were all previous users of the Excellence ${ }^{\infty}$ product, it would be interesting to conduct further research in the general population, or in subjects who have never used hair colouring treatments, and to assess and compare the impact on QoL of different hair colouring formulations and products. While this research was not designed to establish potential superiority this outcome supports the notion that BeautyQoL can be used for product differentiation purposes.

\section{Conclusion}

Hair colouring is used by millions of women and men, who wish to look good, enhance their appearance, self-confidence, and their overall attractiveness. For the first time, using the internationally validated BeautyQoL questionnaire specifically developed to assess cosmetic products and physical appearance, the results of this study scientifically confirm that hair colouring contributes to improving the overall and dimension-specific QoL scores, and the well-being of users worldwide.

These findings further confirm the interest, the robustness and the value of the BeautyQoL specific questionnaire for conducting QoL advanced research to assess and compare cosmetic products and interventions that relate to physical appearance in different populations.

\section{References}

1. Sadick NS (2008) The impact of cosmetic interventions on quality of life. Dermatol Online J 14: 2.

2. Patzer GL (1997) Improving self-esteem by improving physical attractiveness. J Esthet Dent 9: 44-46.

3. Beresniak A, de Linares Y, Krueger GG, Talarico S, Tsutani K, et al. (2012) Validation of a new international quality-of-life instrument specific to cosmetics and physical appearance. Arch Dermatol 148: 1275-1282.

4. Battie C, Verschoore M (2011) Dermatology, cosmetic and well-being. Ann Dermatol Venereol 138: 294-301.

5. Wang KY, Wang KH, Zhang ZP (2011) Health-related quatliy of life and marital quality of vitiligo patients in China. J Eur Acad Dermatol Venereol 25: 429-435.

6. Peuvrel L, Quéreux G, Brocard A, Saint-Jean M, Vallet C, et al. (2012) Evaluation of quality of life after a medical corrective make-up lesson in patients with various dermatoses. Dermatology 224: 374-380.

7. Brain M (2015) How Hair Coloring Works.

8. Ware JE, Sherbourne CD (1992) The MOS 36-item short-form health survey (SF-36). I. Conceptual framework and item selection. Med Care 30: 473-483.

9. Swinburn P, Lloyd A, Boye KS, Edson-Heredia E, Bowman L, et al. (2013) Development of a Disease-Specific Version of the EQ-5D-5L for Use in Patients Suffering from Psoriasis: Lessons Learned from a Feasibility Study in the UK. Value Health 16: 1156-1162.

10. Van Bragt S, van den Bemt L, Thoonen B, Jacobs J, Merkus P, et al. (2014) Validity, reliability and discriminative capacity of an electronic quality of life instrument (Pelican) for childhood asthma in the Netherlands. Qual Life Res 23: 927-938. 